\title{
CONGRUENCES ON MONOIDS OF ORDER-PRESERVING OR ORDER-REVERSING TRANSFORMATIONS ON A FINITE CHAIN
}

\author{
VÍTOR H. FERNANDES \\ Departamento de Matemática, Faculdade de Ciências e Tecnologia, Universidade \\ Nova de Lisboa, Monte da Caparica, 2829-516 Caparica, Portugal \\ e-mail:vhf@fct.unl.pt \\ GRACINDA M. S. GOMES \\ Departamento de Matemática, Faculdade de Ciências, Universidade de Lisboa, \\ 1749-016 Lisboa, Portugal \\ e-mail: ggomes@cii.fc.ul.pt \\ and MANUEL M. JESUS ${ }^{1}$ \\ Departamento de Matemática, Faculdade de Ciências e Tecnologia, Universidade \\ Nova de Lisboa, Monte da Caparica, 2829-516 Caparica, Portugal \\ e-mail:mrj@fct.unl.pt
}

(Received 24 January, 2005; accepted 11 April, 2005)

\begin{abstract}
This paper is mainly dedicated to describing the congruences on certain monoids of transformations on a finite chain $X_{n}$ with $n$ elements. Namely, we consider the monoids $\mathcal{O D}_{n}$ and $\mathcal{P O D} \mathcal{D}_{n}$ of all full, respectively partial, transformations on $X_{n}$ that preserve or reverse the order, as well as the submonoid $\mathcal{P O}$ of $\mathcal{P} \mathcal{O D}_{n}$ of all its

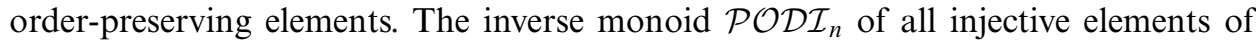
$\mathcal{P O} \mathcal{D}_{n}$ is also considered.

We show that in $\mathcal{P} \mathcal{O}_{n}$ any congruence is a Rees congruence, but this may not happen in the monoids $\mathcal{O D}_{n}, \mathcal{P O D} \mathcal{I}_{n}$ and $\mathcal{P O D} \mathcal{D}_{n}$. However in all these cases the congruences form a chain.
\end{abstract}

2000 Mathematics Subject Classification. 20M20, 20M05, 20 M17.

Introduction and preliminaries. We start by defining the monoids that will be objects of study in this paper. For $n \in \mathbb{N}$, let $X_{n}$ be a finite chain with $n$ elements, say $X_{n}=\{1<2<\cdots<n\}$. As usual, we denote by $\mathcal{P} \mathcal{T}_{n}$ the monoid (under composition) of all partial transformations of $X_{n}$. The submonoid of $\mathcal{P} \mathcal{T}_{n}$ of all full transformations of $X_{n}$ and the (inverse) submonoid of all injective partial transformations of $X_{n}$ are denoted by $\mathcal{T}_{n}$ and $\mathcal{I}_{n}$, respectively.

We say that a transformation $s$ in $\mathcal{P} \mathcal{T}_{n}$ is order-preserving [order-reversing] if $x \leq y$ implies that $x s \leq y s[x s \geq y s]$, for all $x, y \in \operatorname{Dom}(s)$. The following important property of these notions is easy to show: the product of two order-preserving transformations or of two order-reversing transformations is order-preserving and the product of an orderpreserving transformation by an order-reversing transformation is order-reversing.

\footnotetext{
${ }^{1}$ This work was developed within the activities of Centro de Álgebra da Universidade de Lisboa, supported by FCT and FEDER, within project POCTI "Fundamental and Applied Algebra".
} 
Denote by $\mathcal{P} \mathcal{O}_{n}$ the submonoid of $\mathcal{P} \mathcal{T}_{n}$ of all partial order-preserving transformations of $X_{n}$. As usual, $\mathcal{O}_{n}$ denotes the monoid $\mathcal{P} \mathcal{O}_{n} \cap \mathcal{T}_{n}$ of all full transformations of $X_{n}$ that preserve the order. This monoid has been largely studied, namely in $[\mathbf{1}, \mathbf{1 1}$, 13, 14]. The injective counterpart of $\mathcal{O}_{n}$ is the inverse monoid $\mathcal{P O} \mathcal{I}_{n}=\mathcal{P O} \mathcal{O}_{n} \cap \mathcal{I}_{n}$, which is considered, for example, in $[\mathbf{2}, \mathbf{3}, \mathbf{4}, \mathbf{6}, \mathbf{7}, \mathbf{8}]$.

Wider classes of monoids are obtained when we take transformations that either preserve or reverse the order. In this way we get $\mathcal{P O \mathcal { D } _ { n }}$, the submonoid of $\mathcal{P} \mathcal{T}_{n}$ of all partial transformations that preserve or reverse the order. Clearly, we may also consider $\mathcal{O D}_{n}=\mathcal{P O} \mathcal{D}_{n} \cap \mathcal{T}_{n}$ and $\mathcal{P O D} \mathcal{I}_{n}=\mathcal{P O} \mathcal{D}_{n} \cap \mathcal{I}_{n}$, the monoids of all transformations, which preserve or reverse the order, which are full and which are partial and injective, respectively.

The following diagram, with respect to the inclusion relation and in which $\mathbf{1}$ denotes the trivial monoid, clarifies the relationship between these various semigroups.

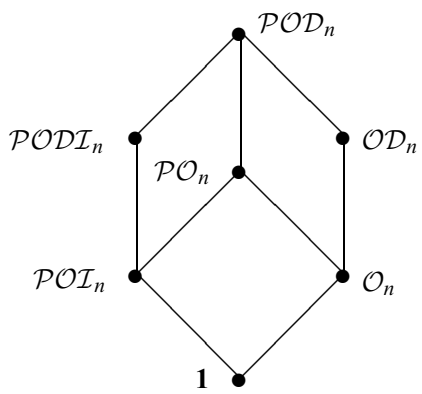

Let $M$ be a monoid. For completion, we recall the definition of the Green's equivalence relations $\mathcal{R}, \mathcal{L}, \mathcal{H}$ and $\mathcal{J}$ : for all $u, v \in M$,

$$
\begin{aligned}
& u \mathcal{R} v \quad \text { if and only if } \quad u M=v M \text {; } \\
& u \mathcal{L} v \text { if and only if } M u=M v \text {; } \\
& u \mathcal{H} v \text { if and only if } u \mathcal{R} v \text { and } u \mathcal{L} v \text {; } \\
& u \mathcal{J} v \text { if and only if } M u M=M v M \text {. }
\end{aligned}
$$

Associated to the Green relation $\mathcal{J}$ there is a quasi-order $\leq \mathfrak{J}$ on $M$ defined by

$$
u \leq_{\mathfrak{g}} v \text { if and only if } M u M \subseteq M v M,
$$

for all $u, v \in M$. Notice that, for every $u, v \in M$, we have $u \mathcal{J} v$ if and only if $u \leq \mathfrak{J} v$ and $v \leq_{\mathfrak{g}} u$. Denote by $J_{u}$ the $\mathcal{J}$-class of the element $u \in M$. As usual, a partial order relation $\leq_{\mathcal{J}}$ is defined on the set $M / \mathcal{J}$ by setting $J_{u} \leq_{\mathcal{J}} J_{v}$ if and only if $u \leq_{\mathcal{J}} v$, for all $u, v \in M$. For $u, v \in M$, we write $u<\mathcal{J} v$ and also $J_{u}<_{\mathfrak{J}} J_{v}$ if and only if $u \leq_{\mathfrak{J}} v$ and $(u, v) \notin \mathcal{J}$.

Given a monoid $M$, we denote by $E(M)$ the set of its idempotents. An ideal of $M$ is a subset $I$ of $M$ such that $M I M \subseteq I$. For convenience, we admit the empty set as an ideal. A Rees congruence of $M$ is a congruence associated to an ideal of $M$ : if $I$ is an ideal of $M$, the Rees congruence $\rho_{I}$ is defined by $(u, v) \in \rho_{I}$ if and only if $u=v$ or $u, v \in I$, for all $u, v \in M$. The rank of a finite monoid $M$ is, by definition, the minimum of the set $\{|X|: X \subseteq M$ and $X$ generates $M\}$. For more details, see, for example, [12].

In this paper, on one hand, we aim to describe the Green's relations on some of the mentioned monoids and to use the descriptions (obtained) to calculate the 
ranks of the respective monoids. These kinds of questions were considered by Gomes and Howie [11] for $\mathcal{O}_{n}$ and $\mathcal{P O}{ }_{n}$, by Fernandes [6] for $\mathcal{P O} \mathcal{I}_{n}$ and by the authors [9] for $\mathcal{P O D} \mathcal{I}_{n}$. It remains to study the monoids $\mathcal{O D}$ and $\mathcal{P O D}$ and this is done in Section 1.

On the other hand, we want to answer a much harder question that consists in describing the congruences. For these monoids, the congruences were known only for $\mathcal{O}_{n}$ and for $\mathcal{P O} \mathcal{I}_{n}$. In fact Aǐzenštat [1], and later Lavers and Solomon [14], showed that in $\mathcal{O}_{n}$ the congruences are exactly the Rees congruences. An analogous result was proved by the first author [6] for the monoid $\mathcal{P O} \mathcal{I}_{n}$.

In Section 2 we also show that the only congruences on $\mathcal{P} \mathcal{O}_{n}$ are the Rees congruences. Section 3 is dedicated to the study of the congruences on $\mathcal{P O D}, \mathcal{P O D} \mathcal{I}_{n}$ and $\mathcal{O} \mathcal{D}_{n}$. In these three cases we prove that there are other congruences besides the Rees congruences, but in all four cases the congruences form a chain.

1. The monoids $\mathcal{O} \mathcal{D}_{n}$ and $\mathcal{P O} \mathcal{D}_{n}$. In this section we aim to describe the Green's relations and to determine the rank of the monoids $\mathcal{O} \mathcal{D}_{n}$ and $\mathcal{P O} \mathcal{D}_{n}$. We show that they have a structure similar to that of the monoids $\mathcal{O}_{n}, \mathcal{P O} \mathcal{O}_{n}, \mathcal{P O \mathcal { I } _ { n }}, \mathcal{P O D \mathcal { I } _ { n }}$ and also of the monoids $\mathcal{P} \mathcal{T}_{n}, \mathcal{T}_{n}$ and $\mathcal{I}_{n}$. In particular, in all of them the $\mathcal{J}$-classes are the sets of all elements with the same rank and, with respect to the partial order $\leq_{\mathcal{g}}$, the corresponding $\mathcal{J}$-quotients are chains. Notice also that these monoids are all regular monoids. Moreover, the monoids $\mathcal{P O} \mathcal{I}_{n}, \mathcal{P O D} \mathcal{I}_{n}$ and $\mathcal{I}_{n}$ are inverse.

In what follows, we must have in mind that an element of $\mathcal{P O} \mathcal{D}_{n}$ is either in $\mathcal{P O}{ }_{n}$ or it is order-reversing. Denote by $\mathcal{P} \mathcal{D}_{n}$ the set of all order-reversing partial transformations of $X_{n}$ and by $\mathcal{D}_{n}$ the subset of $\mathcal{P} \mathcal{D}_{n}$ of all its full transformations. Clearly, $\mathcal{P O} \mathcal{D}_{n}=\mathcal{P} \mathcal{O}_{n} \cup \mathcal{P} \mathcal{D}_{n}$ and so $\mathcal{O D}_{n}=\mathcal{O}_{n} \cup \mathcal{D}_{n}$. Furthermore, $\mathcal{P} \mathcal{O}_{n} \cap \mathcal{P} \mathcal{D}_{n}=$ $\left\{s \in \mathcal{P} \mathcal{T}_{n}:|\operatorname{Im}(s)| \leq 1\right\}$ and so $\mathcal{O}_{n} \cap \mathcal{D}_{n}=\left\{s \in \mathcal{T}_{n}:|\operatorname{Im}(s)|=1\right\}$. It is also easy to show that $E\left(\mathcal{P O} \mathcal{D}_{n}\right)=E\left(\mathcal{P O} \mathcal{O}_{n}\right)$ and so $E\left(\mathcal{O D}_{n}\right)=E\left(\mathcal{O}_{n}\right)$.

Now, consider the following permutation of order two:

$$
h=\left(\begin{array}{ccccc}
1 & 2 & \cdots & n-1 & n \\
n & n-1 & \cdots & 2 & 1
\end{array}\right) .
$$

Clearly, $h$ is an order-reversing full transformation. We showed in [9] that $\mathcal{P O} \mathcal{I}_{n}$ together with $h$ form a set of generators of $\mathcal{P O D} \mathcal{I}_{n}$. Similarly, by just noticing that, given an order-reversing transformation $s$, the product $s h$ is an order-preserving transformation, it is easy to show that $\mathcal{P O} \mathcal{D}_{n}$ is generated by $\mathcal{P O} \cup\{h\}$ and $\mathcal{O D}_{n}$ is generated by $\mathcal{O}_{n} \cup\{h\}$.

Next we prove that $\mathcal{P O D}$ is regular, using the fact that $\mathcal{P O} \mathcal{O}_{n}$ is regular [11]. It is clear that it suffices to show that all the elements of $\mathcal{P} \mathcal{D}_{n}$ are regular. Let $s$ be an order-reversing transformation. Then $s h \in \mathcal{P O} \mathcal{O}_{n}$ and so there exists $s^{\prime} \in \mathcal{P} \mathcal{O}_{n}$ such that $(s h) s^{\prime}(s h)=s h$. Thus, multiplying on the right by $h$, we obtain $s\left(h s^{\prime}\right) s=s$ and so $s$ is a regular element of $\mathcal{P O D}{ }_{n}$. Therefore $\mathcal{P O D}$ is a regular submonoid of $\mathcal{P} \mathcal{T}_{n}$. An analogous reasoning allows us to deduce that $\mathcal{O D}_{n}$ is a regular submonoid of $\mathcal{T}_{n}$ (and of $\left.\mathcal{P} \mathcal{T}_{n}\right)$.

Proposition 1.1. Let $M$ be either the monoid $\mathcal{P O} \mathcal{D}_{n}$ or the monoid $\mathcal{O} \mathcal{D}_{n}$. Let $s$ and $t$ be elements of $M$. Then

1. $s \mathcal{R} t$ if and only if $\operatorname{Ker}(s)=\operatorname{Ker}(t)$;

2. $s \mathcal{L} t$ if and only if $\operatorname{Im}(s)=\operatorname{Im}(t)$;

3. $s \leq_{\mathrm{g}} t$ if and only if $|\operatorname{Im}(s)| \leq|\operatorname{Im}(t)|$. 
Proof. Since $\mathcal{P} \mathcal{O D}_{n}$ and $\mathcal{O} \mathcal{D}_{n}$ are regular submonoids of $\mathcal{P} \mathcal{T}_{n}$, Conditions 1 and 2 follow immediately from well-known results on regular semigroups. (See [12].)

Next, we prove Condition 3. Suppose that $s \leq_{\mathcal{J}} t$. Then there exist $x, y \in M$ such that $s=x t y$, whence $\operatorname{Im}(s) \subseteq \operatorname{Im}(t y)$. Since $|\operatorname{Im}(t y)|=|\operatorname{Im}(t) y| \leq|\operatorname{Im}(t)|$, it follows that $|\operatorname{Im}(s)| \leq|\operatorname{Im}(t y)| \leq|\operatorname{Im}(t)|$.

Conversely, let $s, t \in M$ be such that $|\operatorname{Im}(s)| \leq|\operatorname{Im}(t)|$. First, suppose that $s$ and $t$ are order-preserving transformations. Then, it is well known that $|\operatorname{Im}(s)| \leq|\operatorname{Im}(t)|$ implies $s \leq_{\mathcal{J}} t$ in $\mathcal{P O}_{n}$ (and so in $\mathcal{P O} \mathcal{D}_{n}$ ). If both $s$ and $t$ are full transformations, $|\operatorname{Im}(s)| \leq|\operatorname{Im}(t)|$ also implies $s \leq_{\mathfrak{J}} t$ in $\mathcal{O}_{n}$ (and so in $\mathcal{O D}_{n}$ ). Hence, in this case, $s \leq_{\mathfrak{J}} t$ in $M$. Next, suppose that $s$ is order-preserving and $t$ is order-reversing. Then, as $|\operatorname{Im}(t)|=|\operatorname{Im}(t h)|$ and $s$ and $t h$ are order-preserving, it follows that $s \leq_{\mathcal{J}} t h$ in $M$, by the previous case. Hence there exist $x, y \in M$ such that $s=x(t h) y$. Thus $s \leq_{\mathcal{J}} t$ in $M$. The remaining cases are similar.

It follows, from Condition 3 of the last proposition, that

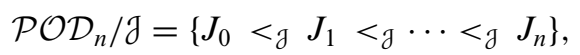

where $J_{k}=\left\{s \in \mathcal{P} \mathcal{O} \mathcal{D}_{n}|| \operatorname{Im}(s) \mid=k\right\}$, for all $0 \leq k \leq n$. Similarly

$$
\mathcal{O D}_{n} / \mathcal{J}=\left\{J_{1}<\mathfrak{J} \cdots<\mathfrak{J} J_{n}\right\},
$$

where $J_{k}=\left\{s \in \mathcal{O D}_{n}|| \operatorname{Im}(s) \mid=k\right\}$, for all $1 \leq k \leq n$.

It is well known that the monoids $\mathcal{O}_{n}$ and $\mathcal{P} \mathcal{O}_{n}$ are aperiodic (i.e. they have trivial $\mathcal{H}$-classes), but that is not the case with $\mathcal{P O} \mathcal{D}_{n}$ and $\mathcal{O D}_{n}$. However in both these cases the situation is not too different as we show next. Let $s \in \mathcal{P O} \mathcal{D}_{n}$. If $s$ has rank less than or equal to 1 , then $s$ is order-preserving and so its $\mathcal{H}$-class (in $\mathcal{P O D} \mathcal{D}_{n}$ and, if $s$ is a full transformation, also in $\mathcal{O D}_{n}$ ) is a singleton. However, if $s$ has rank at least 2, it is easy to show that, with the same kernel and the same image as $s$, there are precisely two elements, $s$ and $t$, say; one is order-preserving and the other is order-reversing. More precisely, if $\operatorname{Im}(s)=\left\{a_{1}<a_{2}<\cdots<a_{m}\right\}$ and $Y_{1}, Y_{2}, \ldots, Y_{m}$ are the classes of $\operatorname{Ker}(s)$ such that $Y_{i} s=\left\{a_{i}\right\}$, for $1 \leq i \leq m$, then the transformation $t \in \mathcal{P O} \mathcal{D}_{n}$ with the same kernel and the same image as $s$ is defined by $Y_{i} t=\left\{a_{m-i+1}\right\}$, for $1 \leq i \leq m$. For example,

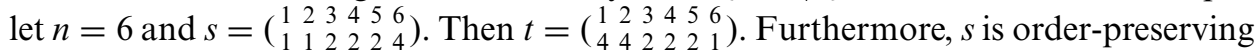
if and only if $t$ is order-reversing, and vice-versa. Summarising, we have the next result.

Proposition 1.2. Let $M$ be either the monoid $\mathcal{P O} \mathcal{D}_{n}$ or the monoid $\mathcal{O D}_{n}$. Let $s$ be an element of $M$ such that $|\operatorname{Im}(s)| \geq 2$. Then $\left|H_{s}\right|=2$. Moreover, the maximal subgroups of the J-classes of $M$ of the elements of rank at least two are cyclic of order two.

When dealing with finite semigroups it is of particular interest to know their size. For example, when we are checking if we have a presentation of a finite monoid $M$, for instance using GAP, knowledge of the cardinal of $M$ is crucial [15, Proposition 3.2.2].

To calculate the cardinals of $\mathcal{P O D} \mathcal{D}_{n}$ and of $\mathcal{O D}_{n}$ we consider the mapping $\varphi$ : $\mathcal{P O}{ }_{n} \longrightarrow \mathcal{P} \mathcal{D}_{n}$ defined by $s \varphi=s h$, for all $s \in \mathcal{P} \mathcal{O}_{n}$. Clearly $\varphi$ is a bijection. Thus we have $\left|\mathcal{P} \mathcal{D}_{n}\right|=\left|\mathcal{P} \mathcal{O}_{n}\right|=\sum_{r=1}^{n}\left(\begin{array}{l}n \\ r\end{array}\right)\left(\begin{array}{c}n+r-1 \\ r\end{array}\right)$, by [11]. On the other hand, the restriction of $\varphi$ to $\mathcal{O}_{n}$ has image $\mathcal{D}_{n}$ and so $\left|\mathcal{D}_{n}\right|=\left|\mathcal{O}_{n}\right|=\left(\begin{array}{c}2 n-1 \\ n-1\end{array}\right)$, by [13]. Now, since $\left|\mathcal{P} \mathcal{O}_{n} \cap \mathcal{P} \mathcal{D}_{n}\right|=$ $\left|\left\{s \in \mathcal{P} \mathcal{T}_{n}:|\operatorname{Im}(s)| \leq 1\right\}\right|=n \sum_{r=1}^{n}\left(\begin{array}{l}n \\ r\end{array}\right)+1$ and $\left|\mathcal{O}_{n} \cap \mathcal{D}_{n}\right|=\left|\left\{s \in \mathcal{T}_{n}:|\operatorname{Im}(s)|=1\right\}\right|=$ $n$, we deduce the following result.

Proposition 1.3. $\left|\mathcal{P O} \mathcal{O D}_{n}\right|=\sum_{r=1}^{n}\left(\begin{array}{c}n \\ r\end{array}\right)\left(2\left(\begin{array}{c}n+r-1 \\ r\end{array}\right)-n\right)-1$ and $\left|\mathcal{O D}_{n}\right|=2\left(\begin{array}{c}2 n-1 \\ n-1\end{array}\right)-n$. 
Naturally, at this point, we should like to compute the rank of these monoids.

As usual, for $x \in \mathbb{R}$, we denote by $\lceil x\rceil$ the least integer greater than or equal to $x$.

Let $n \geq 2$ and $p=\left\lceil\frac{n}{2}\right\rceil$.

First, we consider the monoid $\mathcal{O D}_{n}$. Let

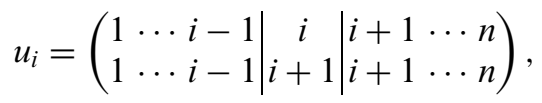

for $1 \leq i \leq n-1$, and recall, from [10], that $\mathcal{O D}_{n}=\left\langle u_{1}, \ldots, u_{n-1}, h\right\rangle$. Now, for $1 \leq$ $j \leq p$, let $z_{j}$ be the (unique) element of $\mathcal{O}_{n}$ (of rank $\left.n-1\right)$ with image $\{1,2, \ldots, j-1$, $j+1, \ldots, n\}$ and kernel defined by the partition $\{\{1\}, \ldots,\{p-j\},\{p-j+1, p-j+2\}$, $\{p-j+3\}, \ldots,\{n\}\}$. It is a routine matter to prove that the following relations hold:

$$
u_{j}=z_{p-j+1} z_{j}, \quad 1 \leq j \leq p,
$$

and

$$
u_{n-j}=h z_{p-j+1} z_{j+1} h, \quad 1 \leq j \leq n-p-1 .
$$

Therefore, we have the following result.

Proposition 1.4. $\mathcal{O D}_{n}=\left\langle z_{1}, \ldots, z_{p}, h\right\rangle$.

Next, we can show that in fact $\mathcal{O D}_{n}$ has rank $p+1$.

THEOREM 1.5. For $n \geq 2$, the monoid $\mathcal{O D}_{n}$ has rank $\left\lceil\frac{n}{2}\right\rceil+1$.

Proof. For $1 \leq i \leq n$, let $D_{i}=\{1,2, \ldots, n\} \backslash\{i\}$. Let $U$ be a set of generators of $\mathcal{O} \mathcal{D}_{n}$. Then, by Proposition 1.4, it suffices to prove that $|U| \geq p+1$. First, notice that there must exist at least one element in $U$ of rank $n-1$. On the other hand, as $h$ is the unique element of rank $n$ that reverses the order, we need to have $h$ in $U$. Observe now that if $f$ is a transformation with image $D_{i}$, for some $1 \leq i \leq n$, then $f h$ has image $D_{n-i+1}$.

Let $\left\{f_{1}, \ldots, f_{k}\right\}$ be the subset of $U$ of all elements of rank $n-1$, for some $k \in \mathbb{N}$. Then, for all $1 \leq i \leq k$, we have $\operatorname{Im}\left(f_{i}\right)=D_{\ell_{i}}$, for some $1 \leq \ell_{i} \leq n$. Thus, if $v \in \mathcal{O} \mathcal{D}_{n}$ is an element of rank $n-1$, we have $v=f f_{i}$ or $v=f f_{i} h$, for some $f \in \mathcal{O D} \mathcal{D}_{n}$ and $1 \leq$ $i \leq k$, and so $\operatorname{Im}(v)=D_{\ell_{i}}$ or $\operatorname{Im}(v)=D_{n-\ell_{i}+1}$. Since there exist $n$ possible images for a transformation of rank $n-1$, the set $\left\{D_{\ell_{1}}, \ldots, D_{\ell_{k}}, D_{n-\ell_{1}+1}, \ldots, D_{n-\ell_{k}+1}\right\}$ has at least (in fact, precisely) $n$ elements, whence $2 k \geq n$. Then $k \geq p$ and so $|U| \geq p+1$, as required.

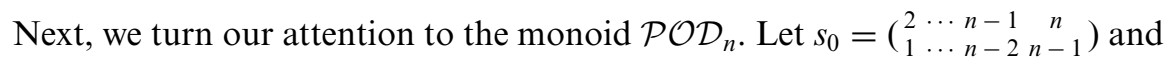

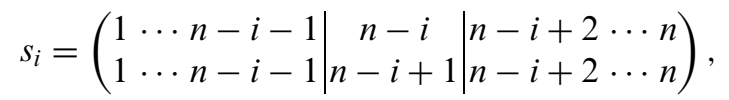

for $i \in\{1,2, \ldots, n-1\}$. In [11], Gomes and Howie proved that $\mathcal{P} \mathcal{O}_{n}=\left\langle s_{0}, \ldots, s_{n-1}\right.$, $\left.u_{1}, \ldots, u_{n-1}\right\rangle$. Since $t h \in \mathcal{P} \mathcal{O}_{n}$ and $t=(t h) h$, for any order-reversing partial transformation $t$, it follows that $\mathcal{P O} \mathcal{D}_{n}=\left\langle s_{0}, \ldots, s_{n-1}, u_{1}, \ldots, u_{n-1}, h\right\rangle$. Let

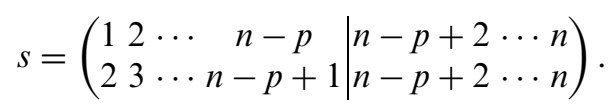


We showed in [9] that

$$
\left\langle s_{1}, \ldots, s_{p-1}, s, h\right\rangle=\left\langle s_{0}, \ldots, s_{n-1}, h\right\rangle\left(=\mathcal{P} \mathcal{O} \mathcal{D} \mathcal{I}_{n}\right)
$$

and so

$$
\mathcal{P O} \mathcal{D}_{n}=\left\langle s_{1}, \ldots, s_{p-1}, s, z_{1}, \ldots, z_{p}, h\right\rangle,
$$

by Proposition 1.4. In this way, we have obtained a generating set of $\mathcal{P O} \mathcal{D}_{n}$ with $n+1$ elements when $n$ is even, and with $n+2$ elements when $n$ is odd. However, if $n$ is odd, we get $z_{2}=h z_{1} h s_{1}$, for $n=3$, and $z_{2}=h z_{1} z_{p} h z_{1} h s_{1} h$, for $n \geq 5$, whence

$$
\mathcal{P O} \mathcal{D}_{n}=\left\langle s_{1}, \ldots, s_{p-1}, s, z_{1}, z_{3}, \ldots, z_{p}, h\right\rangle .
$$

Thus, for an odd integer $n \geq 3$, we also have a generating set of $\mathcal{P O} \mathcal{D}_{n}$ with $n+1$ elements. Hence $\mathcal{P O} \mathcal{D}_{n}$ has rank at most $n+1$. In fact, $n+1$ is exactly its rank.

THEOREM 1.6. For $n \geq 2$, the monoid $\mathcal{P O D}_{n}$ has rank $n+1$.

Proof. It remains to prove that any generating set of $\mathcal{P O} \mathcal{D}_{n}$ has at least $n+1$ elements. Let $U$ be a generating set of $\mathcal{P O} \mathcal{D}_{n}$. In this case, we also must have $h \in U$.

Let $v \in \mathcal{P O D}$ be a transformation of rank $n-1$. Then $v=u z$ or $v=h u z$, for some $u \in U \backslash\{h\}$ and $z \in \mathcal{P O} \mathcal{D}_{n}$. Since $u \neq h$, then $u$ must have rank $n-1$ and so $\operatorname{Ker}(u)=$ $\operatorname{Ker}(v)$ or $\operatorname{Ker}(u)=\operatorname{Ker}(h v)$. Notice that, for $1 \leq j \leq n$, if $v$ is a (full) transformation with kernel defined by the partition $\{\{1\}, \ldots,\{j-1\},\{j, j+1\},\{j+2\}, \ldots,\{n\}\}$, then $h v$ is a (full) transformation with kernel given by the partition $\{\{1\}, \ldots,\{n-j-1\}$, $\{n-j, n-j+1\},\{n-j+2\}, \ldots,\{n\}\}$, for $1 \leq j \leq n-1$. On the other hand, if $v$ is a transformation with kernel given by the partition $\{\{1\}, \ldots,\{j-1\},\{j+1\},\{j+2\}$, $\ldots,\{n\}\}$ (and so $v$ is an injective map), then $h v$ is a transformation with kernel defined by the partition $\{\{1\}, \ldots,\{n-j-1\},\{n-j\},\{n-j+2\}, \ldots,\{n\}\}$ (and so $h v$ also is an injective map). Therefore, $U$ contains at least $\left\lceil\frac{n-1}{2}\right\rceil$ full transformations of rank $n-1$ and at least $\left\lceil\frac{n}{2}\right\rceil$ injective transformations of rank $n-1$, whence $|U| \geq\left\lceil\frac{n-1}{2}\right\rceil+\left\lceil\frac{n}{2}\right\rceil+$ $1=n+1$, as required.

We finish this section by setting some notations and by summarising some properties of the monoids $\mathcal{O} \mathcal{D}_{n}, \mathcal{P O D} \mathcal{I}_{n}$ and $\mathcal{P O D} \mathcal{D}_{n}$ that will be used in the remain two sections.

First, recall that $\mathcal{O D}_{n}=\left\langle\mathcal{O}_{n}, h\right\rangle, \mathcal{P O D} \mathcal{I}_{n}=\left\langle\mathcal{P O} \mathcal{I}_{n}, h\right\rangle$ and $\mathcal{P O} \mathcal{D}_{n}=\left\langle\mathcal{P O} \mathcal{O}_{n}, h\right\rangle$.

Next, let $T \in\left\{\mathcal{O}_{n}, \mathcal{P O} \mathcal{I}_{n}, \mathcal{P O} \mathcal{O}_{n}\right\}$ and $M=\langle T, h\rangle$. Then both $T$ and $M$ are regular monoids (moreover, if $T=\mathcal{P O} \mathcal{I}_{n}$ then $M$ and $T$ are inverse monoids) and $E(M)=$ $E(T)$. Another important property that we shall require is the following: if $s \in M \backslash T$ then $s h, h s \in T$ and the elements $s, s h, h s$ have the same rank.

Remember also that, for the partial order $\leq \mathcal{J}$, the quotients $M / \mathcal{J}$ and $T / \mathcal{J}$ are chains (with $n+1$ elements for $T=\mathcal{P O} \mathcal{I}_{n}$ and $T=\mathcal{P O} \mathcal{O}_{n}$ and with $n$ elements for $\left.T=\mathcal{O}_{n}\right)$. More precisely, if $S \in\{T, M\}$, then

$$
S / \mathcal{J}=\left\{J_{0}^{S}<\mathcal{J} J_{1}^{S}<\mathcal{J} \cdots<\mathcal{J} J_{n}^{S}\right\}
$$

when $T \in\left\{\mathcal{P O} \mathcal{I}_{n}, \mathcal{P O} \mathcal{O}_{n}\right\}$; and

$$
S / \mathcal{J}=\left\{J_{1}^{S}<\mathfrak{J} \cdots<\mathfrak{J} J_{n}^{S}\right\}
$$


when $T=\mathcal{O}_{n}$. Here

$$
J_{k}^{S}=\{s \in S:|\operatorname{Im}(s)|=k\},
$$

with $k$ suitably defined.

For $S \in\{T, M\}$ and $0 \leq k \leq n$, let $I_{k}^{S}=\{s \in S:|\operatorname{Im}(s)| \leq k\}$. Clearly $I_{k}^{S}$ is an ideal of $S$. Since $S / \mathcal{J}$ is a chain, it follows that

$$
\left\{I_{k}^{S}: 0 \leq k \leq n\right\}
$$

is the set of all ideals of $S$.

Finally, observe that $T$ is an aperiodic monoid and that the $\mathcal{H}$-classes of $M$ contained in $J_{k}^{M}$ have precisely two elements (one of them belonging to $T$ and the other belonging to $M \backslash T$ ) when $k \geq 2$. If $k=1$, then such $\mathcal{H}$-classes are trivial.

2. The congruences of the monoid $\mathcal{P \mathcal { O } _ { n }}$. In this section we show that the congruences of $\mathcal{P O} \mathcal{O}_{n}$ are exactly its Rees congruences. We shall make use of the fact that the same happens in $\mathcal{O}_{n}$ and in $\mathcal{P O} \mathcal{I}_{n}[\mathbf{1}, \mathbf{1 4}, \mathbf{6}]$.

First, we prove an easy technical result. Given $s \in \mathcal{P} \mathcal{O}_{n}$, we say that $\bar{s} \in \mathcal{O}_{n}$ is a full $r$-extension of $s$ if $\bar{s}$ extends $s$ (that is $\left.\bar{s}\right|_{\operatorname{Dom}(s)}=s$ ) and $s$ and $\bar{s}$ have the same rank.

Lemma 2.1. Any non-zero element $s \in \mathcal{P} \mathcal{O}_{n}$ has a full r-extension $\bar{s} \in \mathcal{O}_{n}$. by

Proof. Let $\operatorname{Dom}(s)=\left\{i_{1}<\cdots<i_{k}\right\}$, with $1 \leq k \leq n$. For instance, if we define $\bar{s}$

$$
(x) \bar{s}= \begin{cases}\left(i_{1}\right) s, & 1 \leq x \leq i_{1} \\ \left(i_{t}\right) s, & i_{t-1}<x \leq i_{t}, 2 \leq t \leq k ; \\ \left(i_{k}\right) s, & i_{k}<x \leq n\end{cases}
$$

we obtain a full $r$-extension of $s$, as required.

THEOREM 2.2. The congruences of $\mathcal{P O}_{n}$ are exactly its $n+1$ Rees congruences.

Proof. If $n=1$ the result is trivial. Let $n \geq 2$. Let $\rho$ be a congruence of $\mathcal{P O}{ }_{n}$. We aim to find an ideal of $\mathcal{P} \mathcal{O}_{n}$ associated to $\rho$. Let us consider $\bar{\rho}=\rho \cap\left(\mathcal{O}_{n} \times \mathcal{O}_{n}\right)$. There exists $k \in\{1, \ldots, n\}$ such that $\bar{\rho}=\rho_{I_{k}} \mathcal{O}_{n}$, the Rees congruence associated to the ideal $I_{k}^{\mathcal{O}_{n}}$ of $\mathcal{O}_{n}$.

We start by proving that $I_{k}^{\mathcal{P O}} \subseteq 0 \rho$, where 0 denotes the empty map.

If $k=0$, then $I_{k}^{\mathcal{P O}}=\{0\}$ and so, trivially, $I_{k}^{\mathcal{P O}} \subseteq 0 \rho$. Hence, we assume that $k \geq 1$.

Let $c_{1}$ be the constant full transformation of $\mathcal{O}_{n}$ with image $\{1\}$. Clearly $c_{1} \in I_{k}^{\mathcal{O}_{n}}$. Since $n \geq 2$ we can also consider the constant $c_{2}$ with image $\{2\}$. Let $e_{1}$ be the partial identity with domain (and image) $\{1\}$. Then $c_{1}, c_{2} \in I_{k}^{\mathcal{O}_{n}}$ and so $c_{1} \bar{\rho} c_{2}$. Hence $c_{1} \rho c_{2}$. Now $c_{1}=c_{1} e_{1} \rho c_{2} e_{1}=0$. Thus $c_{1} \in 0 \rho$.

Next, we take $s \in J_{k}^{\mathcal{P O}}$. Let $\bar{s}$ be a full $r$-extension of $s$ and let $e$ be the partial identity with domain $\operatorname{Dom}(s)$. Then $e \bar{s}=s$ and, $\bar{s} \in J_{k}^{\mathcal{O}_{n}}$. We have $c_{1}$ and $\bar{s}$ both in $I_{k}^{\mathcal{O}_{n}}$ and so $\bar{s} \bar{\rho} c_{1}$, whence $s=e \bar{s} \rho e c_{1} \rho e 0=0$. Therefore $s \in 0 \rho$. As $s$ generates the ideal $I_{k}^{\mathcal{P O}}$ and $0 \rho$ is an ideal of $\mathcal{P} \mathcal{O}_{n}$, it follows that $I_{k}^{\mathcal{P O}} \subseteq 0 \rho$.

Next, we take $\tilde{\rho}=\rho \cap\left(\mathcal{P O} \mathcal{I}_{n} \times \mathcal{P O} \mathcal{I}_{n}\right)$ and $\ell \in\{0,1, \ldots, n\}$ such that $\tilde{\rho}=\rho_{I_{2} \mathcal{P O I _ { n }}}$ and prove that $I_{\ell}^{\mathcal{P O}} \subseteq 0 \rho$. This inclusion is obvious for $\ell=0$. Assume that $\ell \geq 1$. 
Take $s \in J_{\ell}^{\mathcal{P O}_{n}}$. Let $D$ be any transversal of $\operatorname{Ker}(s)$ and let $e$ be the partial identity with domain $D$. Then es $\in J_{\ell}^{\mathcal{P O} \mathcal{I}_{n}}$ and so es $\tilde{\rho} 0$. Let $(e s)^{-1}$ be the inverse (in $\mathcal{P O} \mathcal{I}_{n}$ ) of es. Then

$$
s=s(e s)^{-1} \text { es } \rho s(e s)^{-1} 0=0
$$

and so $s \in 0 \rho$.

Again, as $s$ generates the ideal $I_{\ell}^{\mathcal{P O} \mathcal{O}_{n}}$, we obtain $I_{\ell}^{\mathcal{P O}} \subseteq 0 \rho$.

Our next step consists of showing that $k=\ell$. Since $I_{k}^{\mathcal{P} \mathcal{O I}} \subseteq I_{k}^{\mathcal{P} \mathcal{O}_{n}} \subseteq 0 \rho$, we have

$$
I_{k}^{\mathcal{P O \mathcal { I } _ { n }}} \subseteq 0 \tilde{\rho}=I_{\ell}^{\mathcal{P O} \mathcal{I}_{n}}
$$

whence $k \leq \ell$.

If $\ell=0$ then $\ell \leq k$ and so $\ell=k$. If $\ell \geq 1$, we have $I_{\ell}^{\mathcal{O}_{n}} \subseteq I_{\ell}^{\mathcal{P O}} \subseteq 0 \rho$ and so

$$
I_{\ell}^{\mathcal{O}_{n}} \subseteq c \bar{\rho}=I_{k}^{\mathcal{O}_{n}}
$$

with $c$ any element of $J_{1}^{\mathcal{O}_{n}}$, whence $\ell \leq k$. Thus $k=\ell$.

Before completing the proof of the theorem we notice that given $s \in \mathcal{P} \mathcal{O}_{n}$ there exists $s^{\prime} \in \mathcal{P O} \mathcal{I}_{n}$ such that $s^{\prime}$ is an inverse of $s$. For example, if $D$ is an arbitrary transversal of $\operatorname{Ker}(s)$ we can take the unique element $s^{\prime} \in \mathcal{P O} \mathcal{I}_{n}$ such that $\operatorname{Dom}\left(s^{\prime}\right)=$ $\operatorname{Im}(s)$ and $\operatorname{Im}\left(s^{\prime}\right)=D$.

Now, we show that $\rho=\rho_{I_{k} \mathcal{P O}_{n}}$. So far we have proved that $I_{k}^{\mathcal{P \mathcal { O } _ { n }}} \subseteq 0 \rho$.

Next, take $s, t \in \mathcal{P O} \mathcal{O}_{n}$ such that $s \rho t$ and $s$ has rank greater than $k$; i.e. $s \notin I_{k}^{\mathcal{P O}}$.

Let $s^{\prime} \in \mathcal{P O} \mathcal{I}_{n}$ be an inverse of $s$ and $t^{\prime} \in \mathcal{P O} \mathcal{I}_{n}$ an inverse of $t$. Then $s \rho t$ implies $s^{\prime} s t^{\prime} t \rho s^{\prime} t t^{\prime} t=s^{\prime} t \rho s^{\prime} s$. Now $s^{\prime} s, t^{\prime} t \in \mathcal{P O} \mathcal{I}_{n}$ and so $s^{\prime} s t^{\prime} t \in \mathcal{P O} \mathcal{I}_{n}$. Hence $s^{\prime} s t^{\prime} t \tilde{\rho} s^{\prime} s$ and, as $s^{\prime} s$ has rank greater than $k$, it follows that $s^{\prime} s t^{\prime} t=s^{\prime} s$, whence $s=\left(s t^{\prime}\right) t$. Then $\operatorname{rank}(t) \geq \operatorname{rank}(s)>k$. Similarly, $\operatorname{rank}(s) \geq \operatorname{rank}(t)$ and $\operatorname{rank}(s)=\operatorname{rank}(t)$.

Now, let $D$ be any transversal of $\operatorname{Ker}(s)$ and let $e$ be the partial identity with domain $D$. Then es and $s$ have the same rank. Since es $\rho$ et, from the above we get $\operatorname{rank}(e s)=\operatorname{rank}(e t)$.

On the other hand,

$$
|D|=|\operatorname{Im}(s)|=|\operatorname{Im}(e t)| \leq|\operatorname{Dom}(e t)|=|D \cap \operatorname{Dom}(t)| \leq|D|,
$$

and so $|D|=|D \cap \operatorname{Dom}(t)|$, whence $D \subseteq \operatorname{Dom}(t)$.

As $D$ is an arbitrary transversal of $\operatorname{Ker}(s)$, it follows that $\operatorname{Dom}(s) \subseteq \operatorname{Dom}(t)$. Similarly, $\operatorname{Dom}(t) \subseteq \operatorname{Dom}(s)$ and so $\operatorname{Dom}(s)=\operatorname{Dom}(t)$.

Next, let $\bar{e}$ be a full $r$-extension of the partial identity with domain $\operatorname{Dom}(s)$ $(=\operatorname{Dom}(t))$. Then $\bar{e} s, \bar{e} t \in \mathcal{O}_{n}$ and $s, t, \bar{e} s$ and $\bar{e} t$ have the same rank (greater than $k$ ). Since $\bar{e} s \rho \bar{e} t$, we have $\bar{e} s \bar{\rho} \bar{e} t$ and as $\bar{\rho}=\rho_{I_{k} \mathcal{O}_{n}}$ we get $\bar{e} s=\bar{e} t$. Hence $s=\left.\bar{e} s\right|_{\operatorname{Dom}(s)}=$ $\left.\bar{e} t\right|_{\operatorname{Dom}(t)}=t$.

Thus, we have proved that $\rho=\rho_{I_{k}^{\mathcal{P O}}}$, as required.

3. The congruences of the monoids $\mathcal{O} \mathcal{D}_{n}, \mathcal{P} \mathcal{O} \mathcal{D} \mathcal{I}_{n}$ and $\mathcal{P O} \mathcal{D}_{n}$. In this section we focus our attention in the study of the congruences of the monoids with order-reversing elements. In what follows $T \in\left\{\mathcal{O}_{n}, \mathcal{P O} \mathcal{I}_{n}, \mathcal{P O} \mathcal{O}_{n}\right\}$ and $M=\langle T, h\rangle$. 
First, notice that it follows from [1] or [14], from [6] and from the last section, that the set $\operatorname{Con}(T)$ of all congruences of $T$ is $\left\{\rho_{I_{k}^{T}}: 0 \leq k \leq n\right\}$.

Next, observe that, for $1 \leq k \leq n$, we can define a congruence $\pi_{k}$ on $M$ as follows: for all $s, t \in M, s \pi_{k} t$ if and only if

1. $s=t$; or

2. $s, t \in I_{k-1}^{M}$; or

3. $s, t \in J_{k}^{M}$ and $s \mathcal{H} t$.

See [5, Lemma 4.2].

For $0 \leq k \leq n$, denote by $\rho_{k}$ the Rees congruence $\rho_{I_{k}^{M}}$ associated to the ideal $I_{k}^{M}$ of $M$ and by $\omega$ the universal congruence of $M$. Clearly, for $n \geq 2$, we have

$$
1=\pi_{1} \subsetneq \rho_{1} \subsetneq \pi_{2} \subsetneq \rho_{2} \subsetneq \cdots \subsetneq \pi_{n} \subsetneq \rho_{n}=\omega .
$$

Our main result of this section establishes that the congruences above are precisely all congruences of $M$.

THEOREM 3.1. Let $M$ be either the monoid $\mathcal{O D}_{n}$ or the monoid $\mathcal{P O D} \mathcal{I}_{n}$ or the monoid $\mathcal{P O D}_{n}$, with $n \geq 2$. Then $M$ has $2 n$ congruences. In fact,

$$
\operatorname{Con}(M)=\left\{1=\pi_{1}, \rho_{1}, \pi_{2}, \rho_{2}, \ldots, \pi_{n}, \rho_{n}=\omega\right\} .
$$
$\{1, \omega\}$.

Observe that $\operatorname{Con}\left(\mathcal{O D}_{1}\right)=\{1\}$ and if $M \in\left\{\mathcal{P O D} \mathcal{I}_{1}, \mathcal{P O} \mathcal{D}_{1}\right\}$ then $\operatorname{Con}(M)=$

To prove Theorem 3.1 we first present some auxiliary results. We start with two routine facts.

Let $c_{1}, \ldots, c_{n} \in \mathcal{T}_{n}$ be such that $\operatorname{Im}\left(c_{i}\right)=\{i\}$, for all $1 \leq i \leq n$ (i.e. $c_{1}, \ldots, c_{n}$ are the $n$ constant mappings of $\mathcal{T}_{n}$ ). Let $s, t \in \mathcal{T}_{n}$ be such that $c_{i} s=c_{i} t$, for all $1 \leq i \leq n$. Then, it is clear that $s=t$.

A version of this property for partial transformation is the following: let $c_{1}, \ldots, c_{n} \in \mathcal{P} \mathcal{T}_{n}$ satisfying $\operatorname{Dom}\left(c_{i}\right)=\operatorname{Im}\left(c_{i}\right)=\{i\}$, for all $1 \leq i \leq n$ (i.e., the $n$ partial identities of rank one). Given $s, t \in \mathcal{P} \mathcal{T}_{n}$ such that $c_{i} s=c_{i} t$, for all $1 \leq i \leq n$, it also is easy to show that $s=t$.

In what follows, $c_{1}, \ldots, c_{n}$ denote the constant mappings of $\mathcal{T}_{n}$ when $M=\mathcal{O D}_{n}$, and the partial identities of rank one of $\mathcal{P} \mathcal{T}_{n}$ when $M=\mathcal{P O D} \mathcal{I}_{n}$ or $M=\mathcal{P O} \mathcal{D}_{n}$.

Notice that in all cases $c_{1}, \ldots, c_{n} \in T$.

Let $\rho$ be a congruence of $M$ and consider $\bar{\rho}=\rho \cap(T \times T)$. Then $\bar{\rho}$ is a Rees congruence of $T$ and so there exists $0 \leq k \leq n$ such that $\bar{\rho}=\rho_{I_{k}^{T}}$.

This notation will be used in the next lemmas.

LEMMA 3.2. If $k=0$ then $\rho=1$.

Proof. First notice that $k=0$ if and only if $\bar{\rho}=1$. Now, it is clear that for all $1 \leq i \leq n$ and $s \in M$ we have $c_{i} s \in T$. (In fact, $c_{i} s$ is either a constant map of image $\{(i) s\}$ or the empty map.) Let $s, t \in M$ be such that $s \rho t$. Then for all $i$, we get $c_{i} s \rho c_{i} t$ and, since $c_{i} s, c_{i} t \in T$, we have $c_{i} s \bar{\rho} c_{i} t$. Hence $c_{i} s=c_{i} t$, for all $1 \leq i \leq n$. Therefore $s=t$ and so $\rho=1$, as required.

From now on, we consider $k \geq 1$.

LEMMA 3.3. $\rho_{k} \subseteq \rho$.

Proof. It suffices to show that $s \rho t$, for all $s, t \in I_{k}^{M}$ and so we consider $s, t \in I_{k}^{M}$. 
If $s, t \in T$ then $s, t \in I_{k}^{T}$ and so $s \bar{\rho} t$, whence $s \rho t$.

If $s, t \in M \backslash T$ then $s h, t h \in T$ and so $s h, t h \in I_{k}^{T}$. Thus $s h \bar{\rho}$ th, whence $s h \rho t h$. Then $s=\operatorname{shh} \rho$ thh $=t$.

Finally, suppose that $s \in M \backslash T$ and $t \in T$. Then $h s \in I_{k}^{T}$ and so $h s \bar{\rho} c_{1}$, since $c_{1} \in I_{k}^{T}$. Hence $h s \rho c_{1}$. Thus $s=h h s \rho h c_{1}$. Since $h c_{1} \in I_{k}^{T}$ (in fact $h c_{1}$ is a constant map), we also have $h c_{1} \bar{\rho} t$, whence $h c_{1} \rho t$. Then $s \rho t$, as required.

LeMmA 3.4. Let $s, t \in M$ be such that $s \rho t$. Then $|\operatorname{Im}(s)|>k$ if and only if $|\operatorname{Im}(t)|>k$.

Proof. We prove that $|\operatorname{Im}(s)|>k$ implies $|\operatorname{Im}(t)|>k$. The converse is analogous.

(1) If $s, t \in T$ then $s \bar{\rho} t$. Since $s \notin I_{k}^{T}$, we have $s=t$, whence $|\operatorname{Im}(t)|>k$.

(2) If $s, t \in M \backslash T$ then $s h$, $t h \in T$ and $s h \rho$ th. Since $|\operatorname{Im}(s h)|=|\operatorname{Im}(s)|>k$, by the previous case we have $s h=t h$, whence $s=t$ and so $|\operatorname{Im}(t)|>k$.

(3) Now, consider $s \in T$ and $t \in M \backslash T$. If $|\operatorname{Im}(t)| \leq k$ then $t \rho_{k} c_{1}$ and so $t \rho c_{1}$, by Lemma 3.3. Hence $s \rho c_{1}$. By the case (1) we get $s=c_{1}$, and this is a contradiction, for $c_{1}$ has rank one. Then $|\operatorname{Im}(t)|>k$.

(4) Finally, suppose that $s \in M \backslash T$ and $t \in T$. Then $s h \in T$ and $s h \rho t h$. Since $|\operatorname{Im}(s h)|=|\operatorname{Im}(s)|>k$, by the case (1) or by the case (3), we deduce that $|\operatorname{Im}(t)|=$ $|\operatorname{Im}(t h)|>k$, as required.

Given a finite semigroup $S$ and $x \in S$, we denote by $x^{\omega}$ the unique idempotent of the subsemigroup of $S$ generated by $x$.

Lemma 3.5. Let $s, t \in M$ be such that $s \rho t$ and $|\operatorname{Im}(s)|>k$. Then $s \mathcal{H} t$.

Proof. Let $s^{\prime}$ and $t^{\prime}$ be (any) inverses of $s$ and $t$, respectively. Then

$$
\left|\operatorname{Im}\left(s s^{\prime}\right)\right|=\left|\operatorname{Im}\left(s^{\prime} s\right)\right|=|\operatorname{Im}(s)|>k
$$

and, by Lemma 3.4,

$$
\left|\operatorname{Im}\left(t t^{\prime}\right)\right|=\left|\operatorname{Im}\left(t^{\prime} t\right)\right|=|\operatorname{Im}(t)|>k .
$$

Since $s \rho t$, we have $s t^{\prime} \rho t t^{\prime}$, whence $\left(s t^{\prime}\right)^{\omega} \rho t t^{\prime}$ and so $\left(s t^{\prime}\right)^{\omega} \bar{\rho} t t^{\prime}$, as $t t^{\prime},\left(s t^{\prime}\right)^{\omega} \in E(M)=$ $E(T)$. Now, since $\left|\operatorname{Im}\left(t t^{\prime}\right)\right|>k$, it follows that $\left(s t^{\prime}\right)^{\omega}=t t^{\prime}$ and so $\left(s t^{\prime}\right)^{\omega} t=t$. Similarly, $\left(t s^{\prime}\right)^{\omega} s=s$, whence $s \mathcal{R} t$.

On the other hand, we have $t^{\prime} s \rho t^{\prime} t$, whence $\left(t^{\prime} s\right)^{\omega} \rho t^{\prime} t$ and so, as above, we may deduce that $\left(t^{\prime} s\right)^{\omega}=t^{\prime} t$. This implies $t\left(t^{\prime} s\right)^{\omega}=t$. Similarly, $s\left(s^{\prime} t\right)^{\omega}=s$, whence $s \mathcal{L} t$.

Thus $s \mathcal{H} t$, as required.

Lemma 3.6. Let $s, t \in M$ be such that $s \neq t$ and $s \mathcal{H} t$. Then, there exists $z \in T$ such that $|\operatorname{Im}(z s)|=|\operatorname{Im}(s)|-1,|\operatorname{Im}(z t)|=|\operatorname{Im}(t)|-1$ and $(z s, z t) \notin \mathcal{L}$.

Proof. Let $m=|\operatorname{Im}(s)|=|\operatorname{Im}(t)|$. Notice that $s \neq t$ and $s \mathcal{H} t$ imply $m \geq 2$. Moreover, we may suppose, without loss of generality, that $s \in T$ and $t \in M \backslash T$.

Let $i_{1}, i_{2}, \ldots, i_{m} \in\{1, \ldots, n\}$ be such that $\operatorname{Im}(s)=\operatorname{Im}(t)=\left\{i_{1}<i_{2}<\cdots<i_{m}\right\}$.

Let $D_{1}, D_{2}, \ldots, D_{m} \subseteq\{1, \ldots, n\}$ be the classes of $\operatorname{Ker}(s)$ such that $D_{\ell} s=\left\{i_{\ell}\right\}$, $1 \leq \ell \leq m$. As $\operatorname{Ker}(s)=\operatorname{Ker}(t)$, by the previous conditions, $D_{\ell} t=\left\{i_{m-\ell+1}\right\}, 1 \leq \ell \leq m$. 
Let $r$ be a fixed element of $D_{m-1}$. We define a transformation $z \in T$ by

$$
(x) z=\left\{\begin{aligned}
x, & x \in D_{1} \cup \cdots \cup D_{m-2}, \\
r, & x \in D_{m-1}, \\
r, & x \in D_{m} \text { and } M=\mathcal{O D}_{n},
\end{aligned}\right.
$$

(if $M \neq \mathcal{O D}_{n}$ we are considering $\left.\operatorname{Dom}(z)=D_{1} \cup \cdots \cup D_{m-1}\right)$. Then $\operatorname{Im}(z s)=$ $\left\{i_{1}, \ldots, i_{m-1}\right\}$ and $\operatorname{Im}(z t)=\left\{i_{2}, \ldots, i_{m}\right\}$, whence $\operatorname{Im}(z s) \neq \operatorname{Im}(z t)$ (since $\left.m \geq 2\right)$. Thus $(z s, z t) \notin \mathcal{L}$. Moreover, $|\operatorname{Im}(z s)|=|\operatorname{Im}(z t)|=m-1$, as required.

Finally, we prove Theorem 3.1.

Proof of Theorem 3.1. Let $\rho \in \operatorname{Con}(M)$ and $\bar{\rho}=\rho \cap(T \times T) \in \operatorname{Con}(T)$. Let $0 \leq$ $k \leq n$ be such that $\bar{\rho}=\rho_{I_{k}^{T}}$.

By Lemma 3.2, if $k=0$ (i.e. $\bar{\rho}=1$ ) then $\rho=1=\pi_{1}$.

Suppose that $1 \leq k \leq n$. Then $\rho_{k} \subseteq \rho$, by Lemma 3.3.

Let $s, t \in M$ be such that $s \neq t, s \rho t$ and $|\operatorname{Im}(s)|>k$. Then, by Lemma 3.5, we have $s \mathcal{H} t$.

Let $m=|\operatorname{Im}(s)|=|\operatorname{Im}(t)|$. As $m>k \geq 1$, by Lemma 3.6, there exists $z \in T$ such that $|\operatorname{Im}(z s)|=|\operatorname{Im}(z t)|=m-1$ and $(z s, z t) \notin \mathcal{L}$. If we have $m-1>k$, as $z s \rho z t$, by Lemma 3.5, we would get $(z s, z t) \in \mathcal{H}$, and this is a contradiction. Thus $m-1=k$. Therefore $\rho \subseteq \pi_{k+1}$.

Suppose that $\rho_{k} \subsetneq \rho$. In this case, we wish to prove that $\rho=\pi_{k+1}$. Observe that $k<n$ and so there exist $s_{0}, t_{0} \in J_{k+1}^{M}$ such that $s_{0} \neq t_{0}, s_{0} \rho t_{0}$ and $s_{0} \mathcal{H} t_{0}$.

Now, take $s, t \in M$ such that $s \neq t$ and $(s, t) \in \pi_{k+1}$. If $s, t \in I_{k}^{M}$ then $(s, t) \in \rho_{k} \subseteq \rho$, whence $(s, t) \in \rho$. If $s, t \in J_{k+1}^{M}$ and $s \mathcal{H} t$. As a consequence of Green's Lemma [12], there exist $a, b \in M$ such that the map $H_{s_{0}} \longrightarrow H_{s}, x \mapsto b x a$ is a bijection. From $H_{s_{0}}=\left\{s_{0}, t_{0}\right\}$, it follows that $\{s, t\}=H_{s}=\left\{b s_{0} a, b t_{0} a\right\}$. Since $s_{0} \rho t_{0}$, we have $b s_{0} a \rho b t_{0} a$ and so $s \rho t$. We may conclude that $\rho=\pi_{k+1}$. We have proved that $\rho=\rho_{k}$ or $\rho=\pi_{k+1}$. The result follows, as required.

\section{REFERENCES}

1. A. Ya. Aǐzenštat, Homomorphisms of semigroups of endomorphisms of ordered sets, Uch. Zap., Leningr. Gos. Pedagog. Inst. 238 (1962), 38-48 (Russian).

2. D. F. Cowan and N. R. Reilly, Partial cross-sections of symmetric inverse semigroups, Int. J. Algebra Comput. 5 (1995) 259-287.

3. V. H. Fernandes, Semigroups of order-preserving mappings on a finite chain: a new class of divisors, Semigroup Forum 54 (1997), 230-236. $418-433$.

4. V. H. Fernandes, Normally ordered inverse semigroups, Semigroup Forum 58 (1998),

5. V. H. Fernandes, The monoid of all injective orientation preserving partial transformations on a finite chain, Comm. Algebra 28 (2000), 3401-3426.

6. V. H. Fernandes, The monoid of all injective order preserving partial transformations on a finite chain, Semigroup Forum 62 (2001), 178-204.

7. V. H. Fernandes, Semigroups of order-preserving mappings on a finite chain: another class of divisors, Izvestiya VUZ. Matematika 3 (478) (2002) 51-59 (Russian).

8. V. H. Fernandes, Presentations for some monoids of partial transformations on a finite chain: a survey, in Semigroups, Algorithms, Automata and Languages, (eds. Gracinda M. S. Gomes \& Jean-Éric Pin \& Pedro V. Silva), (World Scientific, 2002), 363-378. 
9. V. H. Fernandes, G. M. S. Gomes and M. M. Jesus, Presentations for some monoids of injective partial transformations on a finite chain, Southeast Asian Bull. Math. 28 (2004), 903-918.

10. V. H. Fernandes, G. M. S. Gomes and M. M. Jesus, Presentations for some monoids of partial transformations on a finite chain, Comm. Algebra 33 (2005), 587-604.

11. G. M. S. Gomes and J. M. Howie, On the ranks of certain semigroups of orderpreserving transformations, Semigroup Forum 45 (1992), 272-282.

12. J. M. Howie, Fundamentals of semigroup theory (Oxford University Press, 1995).

13. J. M. Howie, Product of idempotents in certain semigroups of transformations, Proc. Edinburgh Math. Soc. (2) 17 (1971) 223-236.

14. T. Lavers and A. Solomon, The endomorphisms of a finite chain form a Rees congruence semigroup, Semigroup Forum 59 (1999), 167-170.

15. N. Ruškuc, Semigroup presentations, Ph. D. Thesis, University of St-Andrews, 1995. 